\title{
Strain-Tuned Structural, Mechanical and Electronic Properties of Two-Dimensional Transition Metal Sulfides ZrS2: A First Principles Study1
}

\section{Qi Song}

Southwest Jiaotong University

Xin Liu

Southwest Jiaotong University

Hui Wang ( $\nabla$ wanghui@swjtu.edu.cn )

Southwest Jiaotong University https://orcid.org/0000-0002-7913-2254

\section{Xiaoting Wang}

Southwest Jiaotong University

\section{Yuxiang Ni}

Southwest Jiaotong University

\section{Hongyan Wang}

Southwest Jiaotong University

\section{Research Article}

Keywords: Zirconium Disulfide, Mechanical anisotropy, Stress-tuned bandgap, Carrier mobility, Optical absorption coefficient

Posted Date: September 20th, 2021

DOl: https://doi.org/10.21203/rs.3.rs-810582/v1

License: (c) (1) This work is licensed under a Creative Commons Attribution 4.0 International License. Read Full License 


\title{
Strain-Tuned Structural, Mechanical and Electronic Properties of Two- dimensional transition metal sulfides $\mathrm{ZrS}_{2}$ : A First Principles Study ${ }^{1}$
}

\author{
Qi Song ${ }^{1}$, Xin Liu ${ }^{1}$, Hui Wang ${ }^{1, *}$, Xiaoting Wang ${ }^{1}$, Yuxiang $\mathrm{Ni}^{1}$, Hongyan Wang ${ }^{1}$ \\ ${ }^{1}$ School of Physical Science and Technology, Southwest Jiaotong University, \\ Chengdu 611756, P.R. China
}

\begin{abstract}
Two-dimensional semiconductor material zirconium disulfide $\left(\mathrm{ZrS}_{2}\right)$ monolayer is a new promising material with good prospects for nanoscale applications. Recently, a new zirconium disulfide $\left(\mathrm{ZrS}_{2}\right)$ monolayer with a space group of 59_Pmmn has been successfully predicted. Using first-principles calculations, this new monolayer $\mathrm{ZrS}_{2}$ structure is obtained with stable indirect band gaps of $0.65 \mathrm{eV}$ and $1.46 \mathrm{eV}$ at the DFTPBE (HSE06) functional levels, respectively. Strain engineering studies on $\mathrm{ZrS}_{2}$ monolayer show effective band gap modulation. The bandgap shows a linear regularity from narrow to wide under applied stresses (strain ranged from $-6 \%$ to $+8 \%$ ). Young's modulus of elasticity of $\mathrm{ZrS}_{2}$ rectangular cells along the tensile directions ( $x$-axis and $y$-axis) is $83.63(\mathrm{~N} / \mathrm{m})$ and $63.61(\mathrm{~N} / \mathrm{m})$ with Poisson's ratios of 0.09 and 0.07 , respectively. The results of carrier mobility show that the electron mobility along the $y$ axis can reach $1.32 \times 10^{3} \mathrm{~cm}^{2} \mathrm{~V}^{-1} \mathrm{~s}^{-1}$. Besides, the order of magnitude of the light absorption coefficient in the ultraviolet spectral region is calculated to reach $2.0 \times 10^{5} \mathrm{~cm}^{-}$ 1 for $\mathrm{ZrS}_{2}$ monolayers. Moreover, by regulating the bandgap under stress, some bandgaps of the stretched energy band exceed the free energy of $1.23 \mathrm{eV}$ and possess a suitable energy band edge position. The results indicates that the new two-dimensional Pmmn- $\mathrm{ZrS}_{2}$ monolayer is a potential material for photovoltaic devices and photocatalytic water decomposition.
\end{abstract}

KEYWORDS: Zirconium Disulfide; Mechanical anisotropy; Stress-tuned bandgap; Carrier mobility; Optical absorption coefficient

\footnotetext{
* Corresponding author: Hui Wang. E-mail: wanghui@swjtu.edu.cn 


\section{INTRODUCTION}

Since the preparation of graphene[1] by laboratory exfoliation in 2004, twodimensional materials have become a popular research area in the last decade or so. Currently, two-dimensional materials have great research potential in the direction of electronic components due to their inherent lightweight (low-dimensional) and highperformance (quantitative) characteristics. Besides, low-dimensional materials such as the hexagonal structure of graphene[2, 3] or one-dimensional carbon nanotubes have shown excellent performance in electronics, mechanics, and optics, prompting more indepth research[4]. Of course, the two-dimensional materials themselves have some defects, such as the inherent zero bandgap of graphene, which greatly limit their practical application value. Therefore, the search for new 2D materials toward more directions and the analysis of their electronic, mechanical as well as optical properties create possibilities for improving the performance of 2D materials[5-9].

Research on transition metal disulfides began with bulk materials[10] in the fields of electronics, optics, mechanics, chemistry, and catalysis. In recent years, the direct bandgap of the material's elemental family, unique electronic structure, and high species diversity have led to increasing investment in related research[11-15]. Two-dimensional Transition-Metal Dichalcogenides (TMDCs) have a chemical structure in the form of $\mathrm{MX}_{2}$, forming a monolayer compound with multiple atomic layers, known as a pincer or sandwich structure. Most of these compounds have semiconductor properties. In recent years, typical two-dimensional semiconductor materials based on TMD-based monolayers, such as $\operatorname{MoX}_{2}[16,17]$ and $\mathrm{WX}_{2}[18]$, have shown great potential for applications in optoelectronic devices due to their inherent wide bandgap and excellent electronic properties.

In 2011, an experimental synthesis of a new two dimensional TMD, the $\mathrm{ZrS}_{2}$ monolayer has been reported by Zeng et al. [9] In 2014, Li et al. [19] systematically explored the elastic, electronic and optical properties of a $\mathrm{D}_{3 \mathrm{~d}}$ point-group symmetric $\mathrm{ZrS}_{2}$ monolayer using properties of the density functional theory. They showed that $2 \mathrm{D}$ $\mathrm{ZrS}_{2}$ is an indirect semiconductor material indicates a band gap of $1.12 \mathrm{eV}(1.93 \mathrm{eV})$ 
calculated by DFT-PBE (HSE06) method which can be modified by imposing an external strain. Recently, a new two-dimensional orthorhombic structure of monolayer $\mathrm{ZrS}_{2}[20]$ with a space group of 59_Pmmn was theoretically proposed and researchers calculated this new two-dimensional structure with a tunable indirect band gap, which we will subsequently investigated in more depth.

In this work, we systematically investigated the electronic, mechanical and optical properties of the new 2D orthorhombic structure of $\mathrm{ZrS}_{2}$ monolayer with a space group of 59_Pmmn by first principles calculations, in which the carrier mobility of $\mathrm{ZrS}_{2}$ monolayers is further calculated by strain regulation. An applied strain offers a novel way of modifying the band gaps of $\mathrm{ZrS}_{2}$ monolayer over a wide range. The energy band (PBE and HSE06 calculations) under the stress of the deformation from $-6 \%$ to $+8 \%$ were obtained by strain engineering to fit and calculate the monolayer $\mathrm{ZrS}_{2}$ transformation situation, and then the carrier mobility of electrons and holes in different directions ( $x$-axis and $y$-axis) were further obtained by using the elastic constants. The optical absorption coefficients were proposed, and the energy values at the edges of the energy band after subtracting the vacuum energy level were larger than the water oxidation, which predicts that monolayer $\mathrm{ZrS}_{2}$ has a good potential application for photocatalytic water decomposition.

\section{METHODS}

The First-principle calculations are performed using the plane wave technique in the Vienna ab initio simulation Package (VASP) [21]. The ion-electron interactions are described by the projector-augmented-wave (PAW) pseudopotentials[22, 23]. The electron exchange-correlation function is treated using the generalized gradient approximation (GGA) in the form proposed by Perdew, Burke, and Ernzehof (PBE) [21, 24]. In all calculations, the energy cutoff for the plane wave is set to $550 \mathrm{eV}$. The convergence threshold is set to $10^{-6} \mathrm{eV}$ of energy. When the magnitude of the force acting on each atom in the cell is reduced to less than $0.001 \mathrm{eV} / \AA$, we consider that the atoms in the cell have reached a position of complete relaxation. A thicker $20 \AA$ vacuum 
layer was added along the z-direction to avoid the effect of interactions with neighboring layers. A $17 \times 17 \times 1$ Monkhorst-Pack $k$-point grid is used for geometric optimization and calculation of electronic properties.

\section{RESULTS AND DISCUSSION}

\subsection{Geometric construction}

(a)

(b)
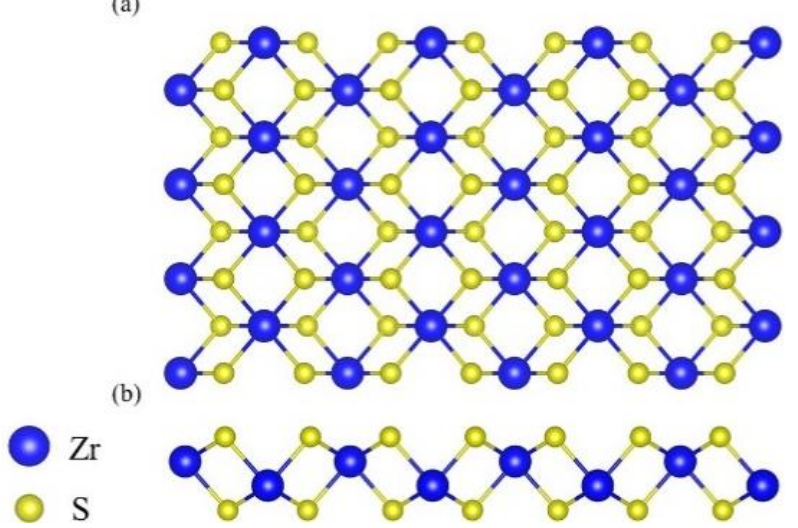

Fig.1 The atomic structure of $\mathrm{ZrS}_{2}$, Top (a) and side (b) views of the unstressed optimized cell structure. Blue and yellow balls represents $\mathrm{Zr}$ and $\mathrm{S}$ atoms, respectively.

Table 1 The calculated lattice parameters (lattice constant $a, b$; bond length for Zr-S; Atomic layer S-S and Zr-S distance; angles of Zr-S-Zr, SZr-S).

\begin{tabular}{|c|c|c|c|c|c|c|c|}
\hline Unit cell type & $\begin{array}{l}a \\
(\AA)\end{array}$ & $\begin{array}{l}b \\
(\AA)\end{array}$ & $\begin{array}{l}\text { lZr-S } \\
(\AA)\end{array}$ & $\begin{array}{l}\triangle_{\mathrm{S}-\mathrm{S}} \\
(\AA)\end{array}$ & $\begin{array}{l}\triangle_{\mathrm{Zr}-\mathrm{S}} \\
(\AA)\end{array}$ & $\angle \mathrm{Zr}-\mathrm{S}-\mathrm{Zr}$ & $\angle \mathrm{s}-\mathrm{Zr}-\mathrm{S}$ \\
\hline \multirow[t]{4}{*}{$\mathrm{ZrS}_{2}$} & 3.67 & 6.49 & 2.55 & 2.89 & 0.97 & $96.71^{\circ}$ & $83.29^{\circ}$ \\
\hline & & & 2.60 & & & $89.91^{\circ}$ & $82.42^{\circ}$ \\
\hline & & & & & & & $132.61^{\circ}$ \\
\hline & & & & & & & $74.10^{\circ}$ \\
\hline
\end{tabular}

The optimization results of the monolayer Pmmn-ZrS 2 structure are shown in Fig. 1 and Table 1. The geometric structure of the form top and side views of $\mathrm{ZrS}_{2}$ is presented in Fig.1(a) and (b). As shown in Figure 1, in the $\mathrm{ZrS}_{2}$ monolayer, the zirconium and sulfur atoms are arranged with a pleated monolayer structure each $\mathrm{Zr}$ atom forms four $\mathrm{Zr}-\mathrm{S}$ bonds with the four adjacent $\mathrm{S}$ atoms. In the top view, $\mathrm{ZrS}_{2}$ shows 
a graphite-like layered structure with zirconium and sulfur atoms forming a fastened hexagonal network. While from the side view, the layered structure follows the S-Zr-S sandwich. Zirconium atoms are not aligned neatly but are arranged in a wavy layer on the middle atomic plane.

As shown in Table 1, the two dimensional Pmmn- $\mathrm{ZrS}_{2}$ structure with lattice parameters of $a=3.67 \AA, b=6.49 \AA$ was obtained. In the $\mathrm{ZrS}_{2}$ monolayer, the zirconium atom is $2.55 \AA$ away from one of the sulfur atoms and $2.60 \AA$ away from the other sulfur atom. The vertical distance between the two layers of sulfur atoms is $2.89 \AA$, and the vertical distance between the sulfur atomic layer and the nearest zirconium atomic layer is $0.97 \AA$. The $\mathrm{Zr}-\mathrm{S}-\mathrm{Zr}$ angle has two different values of $96.71^{\circ}$ and $89.91^{\circ}$, and the $\mathrm{S}-\mathrm{Zr}-\mathrm{S}$ angle has four different values of $83.29^{\circ}, 82.42^{\circ}, 132.61^{\circ}$ and $74.10^{\circ}$, which is consistent with the previous research[20].

\subsection{Energy band and density of states of unstressed $\mathrm{ZrS}_{2}$}

(a)

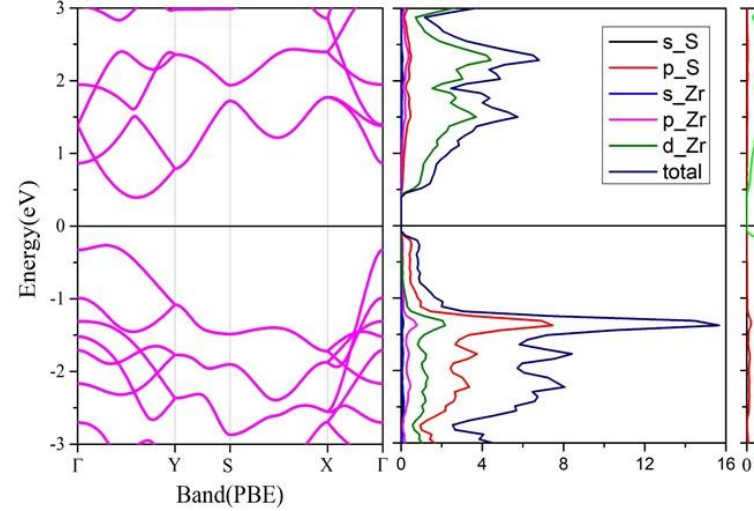

(b)

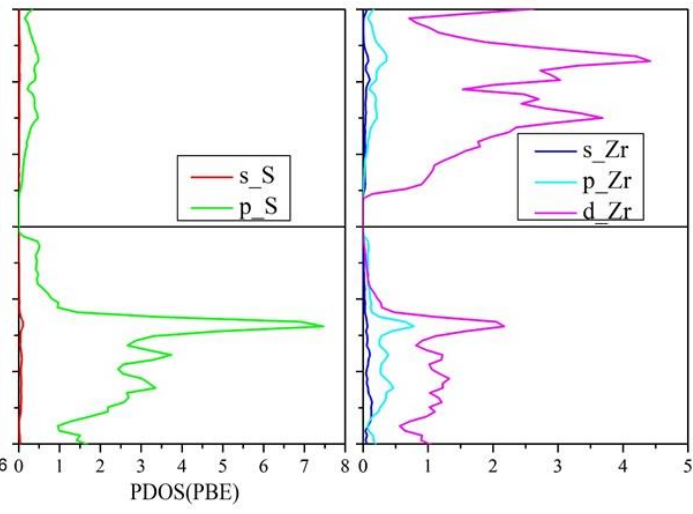

Fig.2 The PBE band structure (a) and PBE partial density of states PDOS (b) of unstressed $\mathrm{ZrS}_{2}$ monolayer.

To gain insight into possible electronic properties of $P m m n-\mathrm{ZrS}_{2}$ in band structure, the band structure and the density of states of this monolayer are computed (Fig.2). According to our calculation, the Pmmn-ZrS $\mathrm{ZS}_{2}$ monolayer shows a semiconducting behavior with a quasi direct band gap of $0.65 \mathrm{eV}$ calculated by DFTPBE method. Due to the fact that DFT-PBE method almost underestimates the band gap, by HSE06 hybrid functional the band structure of the Pmmn- $\mathrm{ZrS}_{2}$ monolayer was recomputed, where a modified band of about $1.46 \mathrm{eV}$ was obtained, which is consistent 
with the previous research results $(0.53 \mathrm{eV} / 1.53 \mathrm{eV}$ for PBE and HSE06 method, respectively)[20]. And the valence band maximum (VBM) and conduction band minimum $(\mathrm{CBM})$ located between the high symmetry points $\mathrm{Y}$ and $\Gamma$.

As shown in Figure 2, The conduction band minimum (CBM) is mainly from the contribution of $\mathrm{Zr}$ atoms, while the valance band maximum (VBM) mainly comes from the joint contribution of $\mathrm{Zr}$ atoms and $\mathrm{S}$ atoms. It is clear from the partial density of states (PDOS) calculations that the CBM is mainly dominated by the d orbitals of the $\mathrm{Zr}$ atom, with contributions from other sub-orbital and various sub-orbital of the $\mathrm{S}$ atom. In contrast, the VBM is mainly dominated by the $\mathrm{d}$ orbital of the $\mathrm{Zr}$ atom and the $\mathrm{p}$ orbital of the S atom after hybridization together, with the other orbitals of the $\mathrm{Zr}$ and S atoms occupying relatively small contributions.

\subsection{Tuning electronic properties by strain}

Table 2 The values of VBM, CBM and bandgap by PBE and HSE06 methods at each strain case.

\begin{tabular}{ccccccccc}
\hline Strains & $b$ & Bandgap & Bandgap & Bandgap & Bandgap & Bandgap & Bandgap & Style \\
$(\%)$ & $\AA$ & $\mathrm{eV}$ & $\mathrm{eV}$ & $\mathrm{eV}$ & $\mathrm{eV}$ & $\mathrm{eV}$ & $\mathrm{eV}$ & \\
& & $\begin{array}{c}\text { (PBE) } \\
\text { uniaxial(x) }\end{array}$ & uniaxial(y) & biaxial & uniaxial(x) & uniaxial(y) & biaxial & \\
\hline-6 & 6.10 & ---- & 0.63 & ---- & 0.98 & 1.40 & 0.73 & indirect \\
-4 & 6.23 & 0.13 & 0.64 & 0.12 & 1.26 & 1.42 & 0.98 & indirect \\
-2 & 6.36 & 0.41 & 0.65 & 0.40 & 1.31 & 1.44 & 1.23 & indirect \\
0 & 6.49 & 0.65 & 0.65 & 0.65 & 1.46 & 1.46 & 1.46 & indirect \\
2 & 6.62 & 0.87 & 0.68 & 0.89 & 1.53 & 1.48 & 1.62 & indirect \\
4 & 6.75 & 1.07 & 0.70 & 1.09 & 1.65 & 1.50 & 1.76 & indirect \\
6 & 6.88 & 1.20 & 0.73 & 1.25 & 1.97 & 1.52 & 1.86 & indirect \\
8 & 7.01 & 1.31 & 0.75 & 1.35 & 2.17 & 1.55 & 1.93 & indirect \\
\hline
\end{tabular}

Because of the efficiency and convenience of applying stress to design and tune the electronic structure properties of two-dimensional materials[25], we further investigated the effect of strain on the band structure of $\mathrm{ZrS}_{2}$ monolayer. We selected 
one of its rectangular cells for the calculations. The PBE and HSE06 band structures of $\mathrm{ZrS}_{2}$ monolayer at each strain case are shown in Fig. S1 in Supporting Information. As shown in Table 2 and Fig.3, for uniaxial tension ( $x$ or $y$ ) and biaxial tension, the band gap of the $\mathrm{ZrS}_{2}$ monolayer decreases or increases when the $\mathrm{ZrS}_{2}$ monolayer is subjected to compressive or tensile strain. The compression or stretching of uniaxial tension $(x$ axis) has more influence on the bandgap than that of uniaxial tension ( $y$-axis). The effect of biaxial compression or stretching on bandgap is similar to that of $x$-axis compression or stretching.

For the compression or stretching of uniaxial tension ( $x$-axis), the reduction of the bandgap is accelerated as the compressive stress increases. Compared with other materials, such as silicene[26], the bandgap of $\mathrm{ZrS}_{2}$ monolayer is more sensitive to changes in compressive stress, and the bandgap of $\mathrm{ZrS}_{2}$ monolayer decreases faster when the compressive stress reaches $6 \%$. When the compressive stress reaches $6 \%$, the bandgap of the $\mathrm{ZrS}_{2}$ monolayer decreases by about $0.5 \mathrm{eV}$. While the tensile stress and its band gap almost have a linear trend, and the bandgap becomes wider and wider with the increase of the tensile stress.

(a)

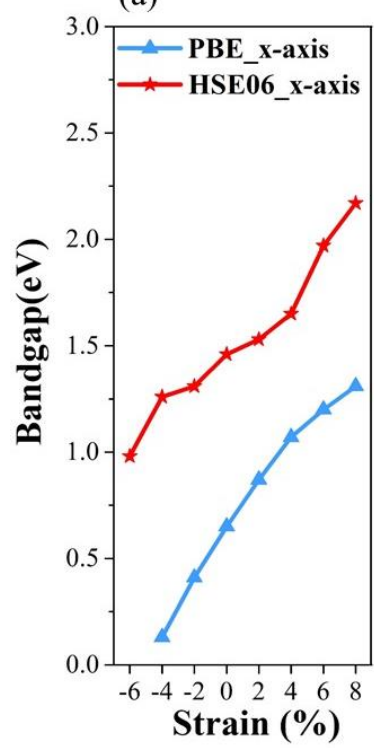

(b)

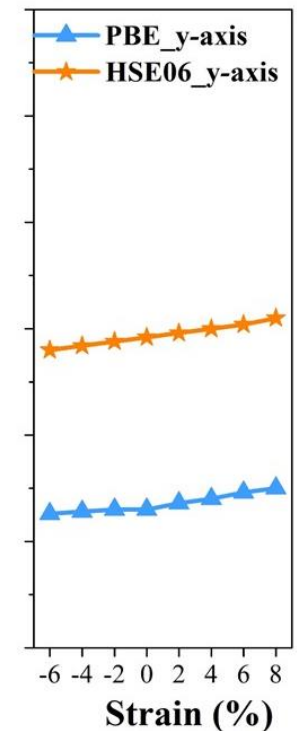

(c)

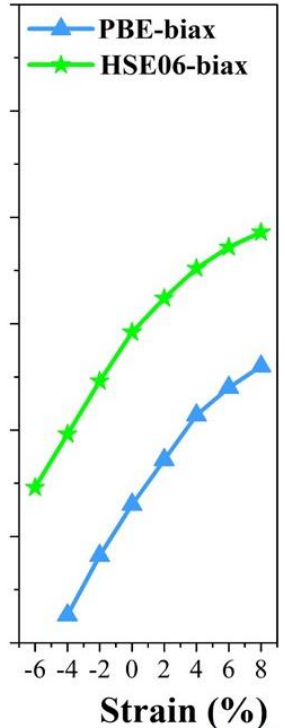

Fig.3 Effect of strain engineering on the bandgap of $\mathrm{ZrS}_{2}$ monolayer. Fig.3(a) shows the bandgaps for uniaxial stretching of the $x$-axis; Fig.3(b) shows the bandgaps for uniaxial stretching of the $y$-axis; Fig.3(c) shows the bandgaps for biaxial stretching 
of the $\mathrm{ZrS}_{2}$ monolayer.

\subsection{Mechanical stability}

\subsubsection{Elastic coefficients}

Table 3 Calculated of 4 independent elastic constants, Young's modulus and Poisson's ratio along the $x$ and $y$ directions.

\begin{tabular}{ccccccc}
\hline Models & $\mathrm{C}_{11}$ & $\mathrm{C}_{22}$ & $\mathrm{C}_{12}$ & $\mathrm{C}_{66}$ & $\mathrm{Y}$ & $v$ \\
& $(\mathrm{~N} / \mathrm{m})$ & $(\mathrm{N} / \mathrm{m})$ & $(\mathrm{N} / \mathrm{m})$ & $(\mathrm{N} / \mathrm{m})$ & $(\mathrm{N} / \mathrm{m})$ & \\
\hline $\mathrm{ZrS}_{2}$ & 84.12 & 63.98 & 5.56 & 2.06 & $83.63(x$-axis $)$ & $0.09(x$-axis $)$ \\
& & & & & $63.61(y$-axis $)$ & $0.07(y$-axis $)$ \\
\hline
\end{tabular}

Based on the elastic solid theory[27], we then calculate the elastic constants of $\mathrm{ZrS}_{2}$ monolayers. For two-dimensional materials, using the standard Voigt notation[28], the strain energy $U$ per unit area is denoted as[29]

$$
U(\varepsilon)=\frac{1}{2} C_{11} \varepsilon_{x x}^{2}+\frac{1}{2} \varepsilon_{y y}^{2}+C_{12} \varepsilon_{x x} \varepsilon_{y y}+2 C_{66} \varepsilon_{x y}^{2}
$$

Where the coefficients $C_{i j}$ (included $C_{11}, C_{12}, C_{22}, C_{66}$ ) are the components of the inplane stiffness tensor, corresponding to the second partial derivative of strain energy concerning strain $\left(C_{i j}=\left[\frac{1}{s_{0}}\left(\partial^{2} U(\varepsilon) / \partial \varepsilon_{i} \partial \varepsilon_{j}\right)\right]\right.$, where $S_{0}$ is the area of the equilibrium unit cell. To guarantee the mechanical stability of the 2D materials, the elastic constants need to satisfy the Born-Huang criteria $\left(C_{11} C_{22}-C_{12}{ }^{2}>0\right.$ and $\left.C_{66}>0\right)$ [30]. All the mechanical parameters listed in Table 3 indicate that the $\mathrm{ZrS}_{2}$ monolayer is mechanically stable. The strain energy of $\mathrm{ZrS}_{2}$ monolayer under uniaxial, shear and biaxial in-plane strain are shown in Fig.S2 in Supporting Information. 


\subsubsection{Anisotropy of Young's modulus and Poisson's ratio}

(a)

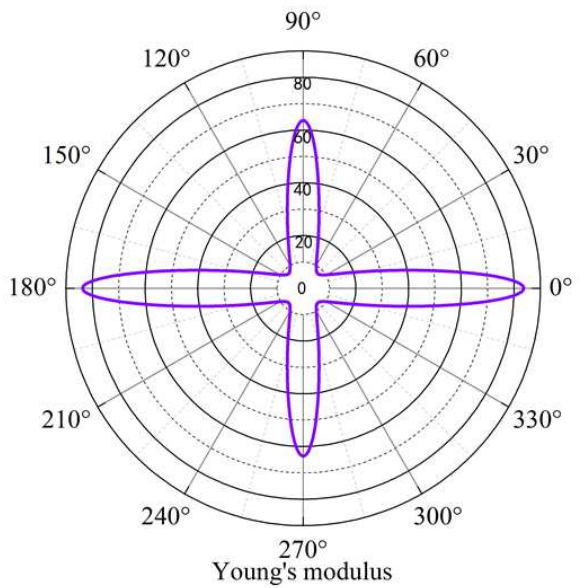

(b)

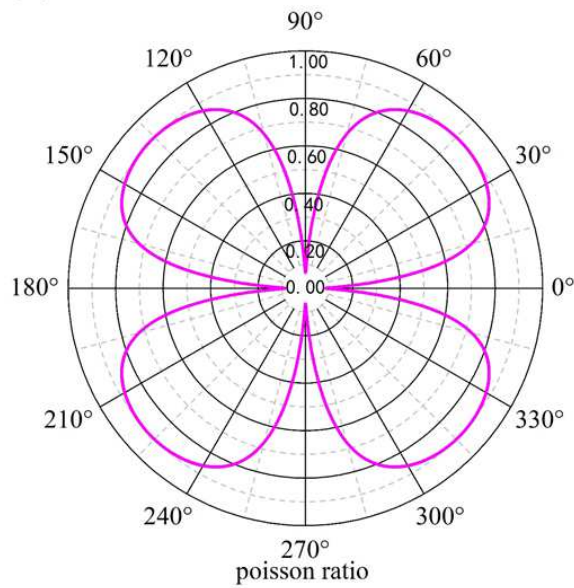

Fig.4 Young's modulus and Poisson's ratio of anisotropic monolayer $\mathrm{ZrS}_{2}$ in polar diagram.

In calculating Young's modulus and Poisson's ratio in different directions, we introduce angles to represent Young's modulus and Poisson's ratio in different directions. They are functions of the direction angles $\theta$, which can be expressed as follows [30-32]:

$$
\begin{aligned}
& Y(\theta)=\frac{C_{11} C_{22}-C_{12}^{2}}{C_{22} \cos ^{4}(\theta)+A \cos ^{2}(\theta) \sin ^{2}(\theta)+C_{11} \sin ^{4}(\theta)} \\
& \nu(\theta)=\frac{C_{12} \cos ^{4}(\theta)-B \cos ^{2}(\theta) \sin ^{2}(\theta)+C_{12} \sin ^{4}(\theta)}{C_{22} \cos ^{4}(\theta)+A \cos ^{2}(\theta) \sin ^{2}(\theta)+C_{11} \sin ^{4}(\theta)}
\end{aligned}
$$

where $\mathrm{A}=\left(C_{11} C_{22}-C_{12}^{2}\right) / C_{66}-2 C_{12}, \mathrm{~B}=C_{11}+C_{22}-\left(C_{11} C_{22}-C_{12}^{2}\right) / C_{66}, C_{i j}$ are calculated elastic coefficients and $\theta$ is the direction angle.

From Fig. 4(a), it is easy to see that Young's modulus in the plane of $\mathrm{ZrS}_{2}$ exhibits large values of $83.63(\mathrm{~N} / \mathrm{m})$ and $63.61(\mathrm{~N} / \mathrm{m})$ in the $x$ - and $y$-axis directions, respectively. The minimum value in the $45.2^{\circ}$ direction is $7.82(\mathrm{~N} / \mathrm{m})$, which indicates that Young's modulus of the $\mathrm{ZrS}_{2}$ monolayer calculated from the tensile strain exhibits anisotropy. From Young's modulus results in each direction, the monolayer $\mathrm{ZrS}_{2}$ is subjected to a certain mechanical response along the direction of the applied stress, while the response to the action in the linear direction of the non-action force is not particularly pronounced. Also, Young's modulus of $\mathrm{ZrS}_{2}$ is relatively small compared to graphene (342 N/m)[28] and $\mathrm{MoS}_{2}(330 \mathrm{~N} / \mathrm{m})[33]$, indicating that the $\mathrm{ZrS}_{2}$ monolayer is more stretchable and flexible, which is important for applications in the direction of electronic devices and flexible materials. 
Figure 4(b) shows the Poisson's ratios in each direction of the $\mathrm{ZrS}_{2}$ monolayer, which is very small in the directions along the $x$-axis and $y$-axis, being approximately 0.09 and 0.07 , respectively. While the Poisson's ratio achieves maximum values in the directions of $45^{\circ}, 135^{\circ}, 225^{\circ}$ and $315^{\circ}$ to the axis, with a maximum value of about 1 . This means that when the material is subjected to a certain degree of tensile deformation, the resistance to deformation along the axis is much weaker than in other directions, especially in the directions of $45^{\circ}, 135^{\circ}, 225^{\circ}$ and $315^{\circ}$ from the axis, where the resistance to deformation is the strongest. The Poisson's ratio of $\mathrm{ZrS}_{2}$ monolayer is somewhat smaller compared to $\mathrm{MoS}_{2}$ monolayer with $v=0.2[34]$, which implies a higher potential for the application of $\mathrm{ZrS}_{2}$ monolayer than $\mathrm{MoS}_{2}$ in strain engineering applications. Graphically, the Poisson's ratio in all directions of the $\mathrm{ZrS}_{2}$ monolayer resembles a four-leaf clover, similar to the butterfly-shaped Poisson's ratio of the Penta$\mathrm{X}_{2} \mathrm{C}$ family the previous report[35].

\subsection{Carrier mobility}

Carrier mobility is usually referred to as the overall movement of electrons and holes within a semiconductor and is an important physical measure of the performance of semiconductor devices[36-40]. Based on the deformation potential theory proposed by Bardeen and Shockley[41], the carrier mobility of two-dimensional materials can be calculated according to the following equation:

$$
\mu_{2 D}=\frac{e \hbar^{3} C_{2 D}}{K_{B} T m^{*} m_{d} E_{1}^{2}}
$$

Where $C_{2 \mathrm{D}}$ is the modulus of elasticity of a uniformly deformed crystal, for $\mathrm{ZrS}_{2}$ monolayer, the calculation results are shown in Fig.S3 in Supporting Information. Where $m^{*}$ is the effective mass in the direction of transmission, $T$ is the temperature, and $k_{\mathrm{B}}$ is the Boltzmann constant. $E_{1}$ represents the deformation potential constant of the electrons located at the top of the valence band (VBM) or clustered at the bottom of the conduction band (CBM) along the direction of transmission, determined by the equation $E_{1}=\Delta E /\left(\Delta l / l_{0}\right)$, where $\Delta E$ is the change in energy of the CBM or VBM under compression or tensile strain, the calculation results of $\Delta E$ are shown in Fig.S4 in Supporting Information. $l_{0}$ is the energy change in the direction of transmission of the 
lattice constant, $\Delta l$ is the deformation of $l_{0} . m_{\mathrm{d}}$ is the average effective mass of the carriers, defined by the following equation: $m_{d}=\sqrt{m_{x}^{*} m_{y}^{*}}$. Based on fitting parabolic functions of the VBM and CBM locations, the carrier effective mass of the $\mathrm{ZrS}_{2}$ can be obtained from the following equation:

$$
m^{*}=\hbar^{2}\left[\frac{\partial^{2} E}{\partial^{2} k}\right]^{-1}
$$

Table 4 Calculated deformation-potential constant, 2D in-plane stiffness, effective mass, and carrier mobility along the $x$ and y directions of $\mathrm{ZrS}_{2}$ monolayer at $300 \mathrm{~K}$.

\begin{tabular}{cccccccccc}
\hline Models & $\begin{array}{c}\text { Carrier } \\
\text { type }\end{array}$ & $\mathrm{m}_{x} / \mathrm{m}_{0}$ & $\mathrm{~m}_{y} / \mathrm{m}_{0}$ & $\mathrm{C}_{x}{ }^{2 \mathrm{~d}}$ & $\mathrm{C}_{y}^{2 \mathrm{~d}}$ & $\mathrm{E}_{1 x}$ & $\mathrm{E}_{1 y}$ & $\begin{array}{c}\mu_{x}^{2 \mathrm{D}} \\
\left(10^{3} \mathrm{~cm}^{2}\right.\end{array}$ & $\begin{array}{c}\mu_{y}^{2 \mathrm{D}} \\
\left(10^{3} \mathrm{~cm}^{2}\right. \\
\end{array}$ \\
& & & & $(\mathrm{N} / \mathrm{m})$ & $(\mathrm{N} / \mathrm{m})$ & $(\mathrm{eV})$ & $(\mathrm{eV})$ & $\begin{array}{c}\left.\mathrm{V}^{-1} \mathrm{~s}^{-1}\right) \\
\left.\mathrm{V}^{-1} \mathrm{~s}^{-1}\right)\end{array}$ \\
\hline $\mathrm{ZrS}_{2}$ & Electron & 1.22 & 0.45 & 84.72 & 63.98 & 0.96 & 2.27 & 0.42 & 1.32 \\
& Hole & -0.24 & -0.65 & 84.72 & 63.98 & -10.63 & 1.45 & 0.38 & 1.33 \\
\hline
\end{tabular}

Table 4 summarizes all the parameters related to the carrier mobility, which are calculated based on the bottom valence band top of the conduction band along the $x$ and $y$-axis directions, respectively. The carrier mobilities of $\mathrm{ZrS}_{2}$ monolayers along the $x$ - and $y$-axis are calculated to be $420 \mathrm{~cm}^{2} \mathrm{~V}^{-1} \mathrm{~s}^{-1}, 1320 \mathrm{~cm}^{2} \mathrm{~V}^{-1} \mathrm{~s}^{-1}$ for electrons and 380 $\mathrm{cm}^{2} \mathrm{~V}^{-1} \mathrm{~s}^{-1}, 1330 \mathrm{~cm}^{2} \mathrm{~V}^{-1} \mathrm{~s}^{-1}$ for holes, respectively. For $\mathrm{PtS}_{2}$ and $\mathrm{PtSe}_{2}$, the obtained electron mobility along the $x$ direction are $2411.50 \mathrm{~cm}^{2} \mathrm{~V}^{-1} \mathrm{~s}^{-1}$ and $1103.04 \mathrm{~cm}^{2} \mathrm{~V}^{-1} \mathrm{~s}^{-1}$, whereas the hole mobility along the $x$ direction is about $136.20 \mathrm{~cm}^{2} \mathrm{~V}^{-1} \mathrm{~s}^{-1}$ and 217.85 $\mathrm{cm}^{2} \mathrm{~V}^{-1} \mathrm{~s}^{-1}$, respectively. Simultaneously, for $\mathrm{PtS}_{2}$ and $\mathrm{PtSe}_{2}$ the electron mobility along the $y$ direction is $1477.13 \mathrm{~cm}^{2} \mathrm{~V}^{-1} \mathrm{~s}^{-1}$ and $1289.32 \mathrm{~cm}^{2} \mathrm{~V}^{-1} \mathrm{~s}^{-1}$, while the hole mobility along the $y$ direction is $218.30 \mathrm{~cm}^{2} \mathrm{~V}^{-1} \mathrm{~s}^{-1}$ and $1161.70 \mathrm{~cm}^{2} \mathrm{~V}^{-1} \mathrm{~s}^{-1}$,[39] respectively.

Although compared with the maximum carrier mobilities of $4 \times 10^{5} \mathrm{~cm}^{2} \mathrm{~V}^{-1} \mathrm{~s}^{-1}$ for graphene and $200 \mathrm{~cm}^{2} \mathrm{~V}^{-1} \mathrm{~s}^{-1}$ for $\mathrm{MoS}_{2}$, the monolayer $\mathrm{ZrS}_{2}$ has lower carrier mobility than graphene but it still has better carrier mobility. What's more important is that monolayer $\mathrm{ZrS}_{2}$ has the same characteristics as monolayer $\mathrm{PtS}_{2}$ and $\mathrm{PtSe}_{2}$. Large differences in the values of electron and hole carrier mobilities in different directions, which will lead to the rapid migration of photogenerated electrons and holes. And the 
numerical difference between the electron and hole carrier mobilities indicates the anisotropy of hole and electron carriers, which facilitates a more efficient separation of electron-hole pairs of electrons. The difference in mobility between electrons and holes allows the monolayer $\mathrm{ZrS}_{2}$ material can to be used for photocatalytic hydrolysis for a long time, maintaining the photocatalytic activity.

\subsection{Absorption coefficient}

From a practical application point of view, we are very concerned about the optical performance of the $\mathrm{ZrS}_{2}$ monolayer. We have calculated absorption properties based on the dielectric function $\varepsilon(\omega)=\varepsilon_{1}(\omega)+i \varepsilon_{2}(\omega)$, where $\omega$ is the frequency. The absorption coefficient $\alpha(\omega)$ was calculated using: [42]

$$
\alpha(\omega)=\frac{\sqrt{2 \omega}}{c}\left(\sqrt{\varepsilon_{1}^{2}(\omega)+\varepsilon_{2}^{2}(\omega)}-\varepsilon_{1}(\omega)\right)^{1 / 2}
$$

where $\varepsilon_{1}$ is the real part of the complex dielectric function, which could be obtained from $\varepsilon_{2}$ using the Kramer-Kronig relationship. $\varepsilon_{2}$ is defined as: [43]

$$
\begin{gathered}
\varepsilon_{2}(\omega)=\frac{4 \pi^{2} e^{2}}{\Omega} \lim _{q \rightarrow 0} \frac{1}{q^{2}} \sum_{c, v, \vec{k}} 2 w_{k} \delta\left(\varepsilon_{c k}-\varepsilon_{v k}-\omega\right) \\
\times\left\langle\mu_{c k+e_{a q}} \mid \mu_{v k}\right\rangle\left\langle\mu_{c k+e_{\beta q}} \mid \mu_{v k}\right\rangle^{*}
\end{gathered}
$$

where $\alpha$ and $\beta$ refer to the $x$ and $y$ directions, and $\Omega$ is the volume of the unit cell. The indices $c$ and $v$ refer to the conduction and valence band states, respectively. $\mu_{c k}$ corresponds to an eigenstate with wave vector $k$. 


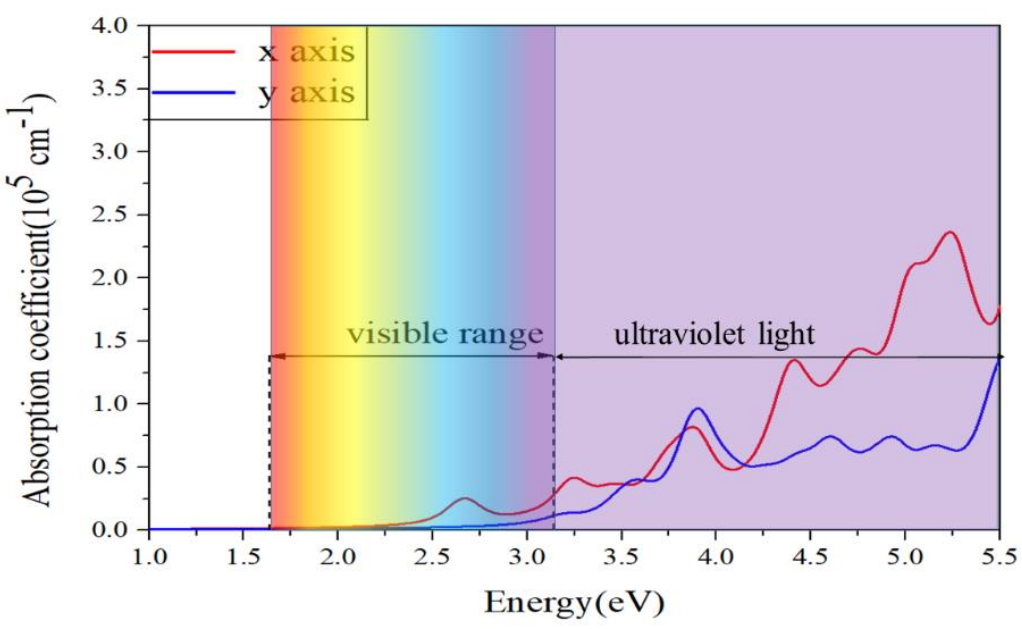

Fig.5 The calculated light absorption spectra for the $\mathrm{ZrS}_{2}$ monolayer.

We calculated the light absorption coefficients of $\mathrm{ZrS}_{2}$ monolayer along different transmission directions ( $x$-axis and $y$-axis). The spectrum transitions from the infrared region to the ultraviolet region and the overall light absorption coefficient of $\mathrm{ZrS}_{2}$ monolayer material in the planar direction along the $x$-axis is generally larger than that of $\mathrm{ZrS}_{2}$ along the planar $y$-axis direction. In the visible regions, the light absorption coefficients along the $x$ - and $y$-axis can reach $0.5 \times 10^{5} \mathrm{~cm}^{-1}$, but it still appear low compared to the UV region. In the UV region, the light absorption coefficient along the $x$-axis can reach more than $2.0 \times 10^{5} \mathrm{~cm}^{-1}$, and the light absorption coefficient along the $y$-axis can also reach more than $1.25 \times 10^{5} \mathrm{~cm}^{-1}$. Compared with the visible region, the $\mathrm{ZrS}_{2}$ monolayer performs well in the UV region, which indicates that $\mathrm{ZrS}_{2}$ monolayer may be a promising material for photovoltaic applications. 


\subsection{Band edge positions of $\mathrm{ZrS}_{2}$ monolayer}

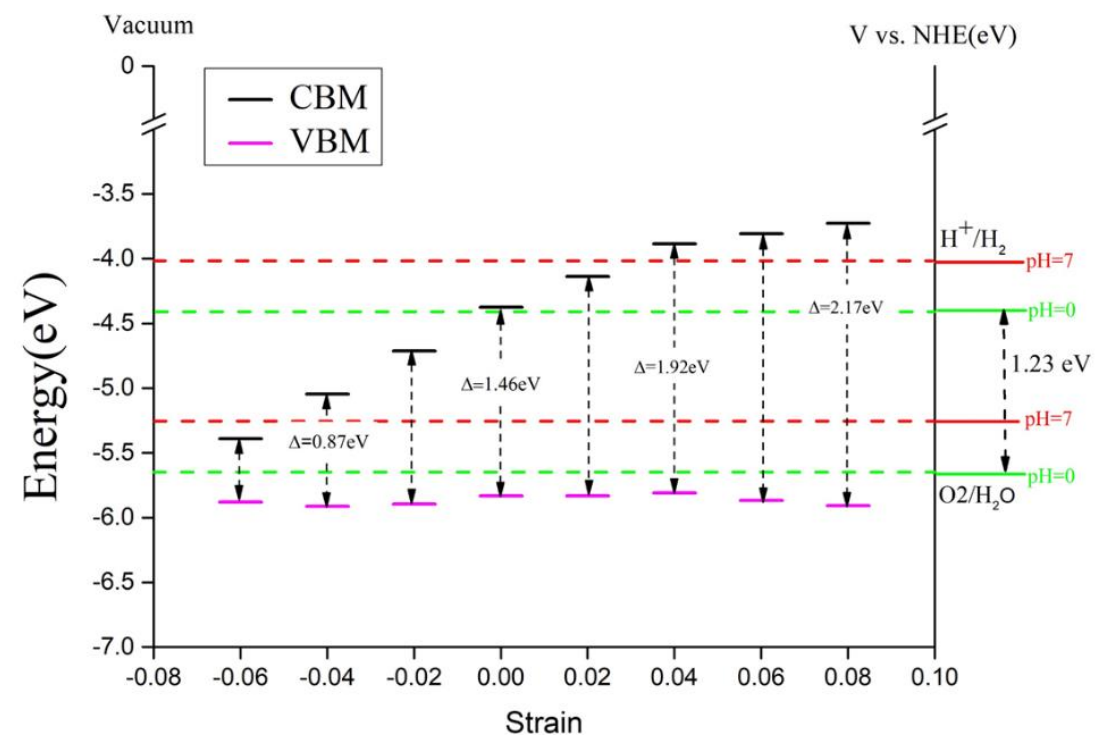

Fig.6 The position of the band edge of the $\mathrm{ZrS}_{2}$ monolayer influenced by strain stretching.

As we know, the most important condition for a two-dimensional semiconductor material to be used for photocatalytic hydrolysis [44-46] is to have a bandgap exceeding a certain forbidden bandwidth, the theoretical value of this bandgap must be greater than the electrolytic voltage of water, $1.23 \mathrm{eV}$. In practice, the $2 \mathrm{D}$ semiconductor should be able to withstand a voltage greater than or equal to $1.6 \mathrm{eV}$ to ensure that the photocatalytic reaction can be carried out in the visible light range. To ensure that the reduction and oxidation of water can occur, the conduction band should lie at a lower potential than the reduction potential of water (at $0 \mathrm{~V} \mathrm{NHE} \mathrm{pH}=0$ ) and the valence band should be higher than the oxidation potential of water.

As shown in Fig.6, we sorted out the position of the band edge of the $\mathrm{ZrS}_{2}$ monolayer after stress conditioning, and the comparison with the redox potential of water shows that the bandgap width of the $\mathrm{ZrS}_{2}$ monolayer $1.46 \mathrm{eV}$ is larger than the electrolytic voltage of water, and we obtained a wider bandgap of the forbidden band $(2.17 \mathrm{eV})$ by the stretching of stress. It is worth noting that the valence band top changes very slightly when stretching the $\mathrm{ZrS}_{2}$ monolayer, considering the efficiency of the actual water decomposition, so the potential of the conduction band bottom cannot be too high. It is easy to see from Fig. 6 that the conduction band bottom and valence band 
low are more symmetrically distributed at the more outer side of the redox potential under the condition of $2 \%$ tensile stress. $\mathrm{ZrS}_{2}$ monolayer is able to regulate the bandgap by stress to reach the potential requirement for decomposition, then which is a promising material for photocatalytic hydrolysis.

\section{CONCLUSIONS}

In summary, we have verified the electronic and mechanical properties of the new configuration Pmmn- $\mathrm{ZrS}_{2}$ monolayer using first-principles calculations, confirming its stability. The bandgap of $0.65 \mathrm{eV}$ was calculated by the PBE method, which largely underestimates the value of the bandgap (compared to the HSE06 method 1.46eV). Then, we applied stress to the Pmmn-ZrS 2 structure, stretching it in different directions from $-6 \%$ to $8 \%$ to modulate the bandgap. For $x$-axis tension, band gap data are obtained from $0 \mathrm{eV}$ to $1.31 \mathrm{eV}$ (PBE method), corresponding to the results of the HSE06 method from $0.98 \mathrm{eV}$ to $2.17 \mathrm{eV}$. With the bang gap dates calculated by the PBE method, we obtained the parameters of the mechanical properties of $\mathrm{ZrS}_{2}$ monolayers, including the elastic constants, Young's modulus, and Poisson's ratio under uniaxial stress conditions. The results show that the elastic constants of the $\mathrm{ZrS}_{2}$ monolayer is relatively smaller than those of other transition metal sulfide groups, the smaller Young's modulus and Poisson's ratio indicate that $\mathrm{ZrS}_{2}$ monolayer is a relatively soft two-dimensional material that can be used as an ideal material for sensors. By calculating the carrier mobility of the $\mathrm{ZrS}_{2}$ monolayer, we are pleased to find that the electron and hole mobilities in the $x$ and $y$ axis are numerically different and have more pronounced differences. The value of carrier mobility along the $y$ direction reaches $1320 \mathrm{~cm}^{2} \mathrm{~V}^{-1} \mathrm{~s}^{-}$

${ }^{1}$. The high carrier mobility indicates that the $\mathrm{ZrS}_{2}$ monolayer is valuable for making photovoltaic materials. By calculating the light absorption coefficients in different directions, the absorption value of the $\mathrm{ZrS}_{2}$ monolayer in the UV region reaches $2.5 \times$ $10^{5}$. In addition, the strain modulation makes it easier for the $\mathrm{ZrS}_{2}$ monolayer to meet the conditions of hydrolytic photocatalysis at $\mathrm{pH}=0$ and $\mathrm{pH}=7$. This study shows that the new two-dimensional Pmmn- $\mathrm{ZrS}_{2}$ monolayer is a potential material for photovoltaic devices and photocatalytic hydrolysis. 


\section{— SUPPORTING INFORMATIONS}

Fig.S1 The PBE and HSE06 band structure of $\mathrm{ZrS}_{2}$ monolayer at each strain case.

Fig.S2 Strain energy of $\mathrm{ZrS}_{2}$ monolayer under uniaxial, shear and biaxial in-plane strain, respectively.

Fig.S3 Fit results for $\mathrm{ZrS}_{2}$ monolayer with different directional elastic constants $C_{2 \mathrm{~d}}$, respectively.

Fig.S4 Deformation potential constant for different directions of $\mathrm{ZrS}_{2}$ monolayer, respectively.

\section{Declarations}

\section{Funding}

We acknowledge financial support from the National Natural Science Foundation of China (Grant No. 11404268, 11774294).

\section{Conflicts of interest}

There are no conflicts of interest to declare.

\section{Availability of data and material}

N/A

\section{Code Availability}

All data, models, and code generated or used during the study appear in the submitted article.

\section{Authors Contribution Statement}

Qi Song performed the data computation, analysis and wrote the first draft of manuscript;

Xin Liu contributed to the data analysis and Program Testing;

Hui Wang contributed to the topic selection, data analysis and manuscript revision;

Xiaoting Wang helped to the manuscript preparation;

Yuxiang Ni helped perform the Program testing;

Hongyan Wang helped perform the constructive discussions.

\section{REFERENCES}

[1] Novoselov KS, Geim AK, Morozov SV, Jiang D, Zhang Y, Dubonos SV, Grigorieva IV, Firsov AA (2004) Electric Field Effect in Atomically Thin Carbon Films. Science 306(5696):666

[2] Hernandez Rosas JJ, Ramirez Gutierrez RE, Escobedo-Morales A, Chigo Anota E (2011) First principles calculations of the electronic and chemical properties of graphene, graphane, and graphene oxide. J Mol Model 17(5):1133-9

[3] Malko D, Neiss C, Vines F, Gorling A (2012) Competition for graphene: graphynes with directiondependent Dirac cones. Phys Rev Lett 108(8):086804 
[4] Balendhran S, Walia S, Nili H, Sriram S, Bhaskaran M (2015) Elemental analogues of graphene: silicene, germanene, stanene, and phosphorene. Small 11(6):640-52

[5] Bhimanapati GR, Lin Z, Meunier V, Jung Y, Cha J, Das S, Xiao D, Son Y, Strano MS, Cooper VR, Liang L, Louie SG, Ringe E, Zhou W, Kim SS, Naik RR, Sumpter BG, Terrones H, Xia F, Wang Y, Zhu J, Akinwande D, Alem N, Schuller JA, Schaak RE, Terrones M, Robinson JA (2015) Recent Advances in Two-Dimensional Materials beyond Graphene. ACS Nano 9(12):11509-11539

[6] Mannix AJ, Zhou X-F, Kiraly B, Wood JD, Alducin D, Myers BD, Liu X, Fisher BL, Santiago U, Guest JR, Yacaman MJ, Ponce A, Oganov AR, Hersam MC, Guisinger NP (2015) Synthesis of borophenes: Anisotropic, two-dimensional boron polymorphs. Science 350(6267):1513

[7] Molle A, Grazianetti C, Tao L, Taneja D, Alam MH, Akinwande D (2018) Silicene, silicene derivatives, and their device applications. Chem Soc Rev 47(16):6370-6387

[8] Vogt P, De Padova P, Quaresima C, Avila J, Frantzeskakis E, Asensio MC, Resta A, Ealet B, Le Lay G (2012) Silicene: compelling experimental evidence for graphenelike two-dimensional silicon. Phys Rev Lett 108(15):155501

[9] Zeng Z, Yin Z, Huang X, Li H, He Q, Lu G, Boey F, Zhang H (2011) Single-layer semiconducting nanosheets: high-yield preparation and device fabrication. Angew Chem Int Ed Engl 50(47):11093-7

[10] Ponce S, Li W, Reichardt S, Giustino F (2020) First-principles calculations of charge carrier mobility and conductivity in bulk semiconductors and two-dimensional materials. Rep Prog Phys 83(3):036501

[11] Beiranvand R (2021) Theoretical investigation of electronic and optical properties of 2D transition metal dichalcogenides $\mathrm{MoX}_{2}(\mathrm{X}=\mathrm{S}, \mathrm{Se}, \mathrm{Te})$ from first-principles. Physica E: Low-dimensional Systems and Nanostructures 126:114416

[12] Li H, Shi Y, Chiu M-H, Li L-J (2015) Emerging energy applications of two-dimensional layered transition metal dichalcogenides. Nano Energy 18:293-305

[13] Ma M, Yao Y, Wu Y, Yu Y (2020) Progress and Prospects of Transition Metal Sulfides for Sodium Storage. Advanced Fiber Materials 2(6):314-337

[14] Oliver SM, Fox JJ, Hashemi A, Singh A, Cavalero RL, Yee S, Snyder DW, Jaramillo R, Komsa H$\mathrm{P}$, Vora PM (2020) Phonons and excitons in $\mathrm{ZrSe}_{2}-\mathrm{ZrS}_{2}$ alloys. Journal of Materials Chemistry C 8(17):5732-5743

[15] Wang QH, Kalantar-Zadeh K, Kis A, Coleman JN, Strano MS (2012) Electronics and optoelectronics of two-dimensional transition metal dichalcogenides. Nat Nanotechnol 7(11):699-712

[16] Cai Y, Zhang G, Zhang YW (2014) Polarity-reversed robust carrier mobility in monolayer MoS(2) nanoribbons. J Am Chem Soc 136(17):6269-75

[17] Lagarde D, Bouet L, Marie X, Zhu CR, Liu BL, Amand T, Tan PH, Urbaszek B (2014) Carrier and polarization dynamics in monolayer MoS2. Phys Rev Lett 112(4):047401

[18] Guo H, Zhang Z, Huang B, Wang X, Niu H, Guo Y, Li B, Zheng R, Wu H (2020) Theoretical study on the photocatalytic properties of $2 \mathrm{D} \mathrm{InX}(\mathrm{X}=\mathrm{S}, \mathrm{Se}) /$ transition metal disulfide $\left(\mathrm{MoS}_{2}\right.$ and $\left.\mathrm{WS}_{2}\right)$ van der Waals heterostructures. Nanoscale 12(38):20025-20032

[19] Li Y, Kang J, Li JB (2014) Indirect-to-direct band gap transition of the $\mathrm{ZrS}_{2}$ monolayer by strain: first-principles calculations. Rsc Advances 4(15):7396-7401

[20] Abutalib MM (2019) A DFT based prediction of a new 2D zirconium disulfide Pmmm-ZrS 2 monolayer: A quasi direct band gap semiconductor. Results in Physics 12:903-907

[21] Kresse G, Furthmüller J (1996) Efficient iterative schemes for ab initio total-energy calculations using a plane-wave basis set. Physical Review B 54(16):11169-11186

[22] Blochl PE (1994) Projector augmented-wave method. Phys Rev B Condens Matter 50(24):17953- 
17979

[23] Kresse G, Joubert D (1999) From ultrasoft pseudopotentials to the projector augmented-wave method. Physical Review B 59(3):1758-1775

[24] Perdew JP, Burke K, Ernzerhof M (1996) Generalized Gradient Approximation Made Simple. Physical Review Letters 77(18):3865-3868

[25] Chen S-B, Zeng Z-Y, Chen X-R, Yao X-X (2020) Strain-induced electronic structures, mechanical anisotropy, and piezoelectricity of transition-metal dichalcogenide monolayer $\mathrm{CrS}_{2}$. Journal Of Applied Physics 128(12):125111

[26] Chen C-H, Li W-W, Chang Y-M, Lin C-Y, Yang S-H, Xu Y, Lin Y-F (2018) Negative-DifferentialResistance Devices Achieved by Band-Structure Engineering in Silicene under Periodic Potentials. Physical Review Applied 10(4):044047

[27] Landau L, Lifshitz E (1986) Theory of Elasticity. Pergammon, Oxford, UK

[28] Andrew RC, Mapasha RE, Ukpong AM, Chetty N (2012) Mechanical properties of graphene and boronitrene. Physical Review B 85(12):125428

[29] Wei Q, Peng XH (2014) Superior mechanical flexibility of phosphorene and few-layer black phosphorus. Applied Physics Letters 104(25):251915

[30] Cadelano E, Palla PL, Giordano S, Colombo L (2010) Elastic properties of hydrogenated graphene. Physical Review B 82(23):235414

[31] Hu X, Mao N, Wang H, Niu C, Huang B, Dai Y (2019) Two-dimensional ferroelastic topological insulator with tunable topological edge states in single-layer $\mathrm{ZrAsX}(\mathrm{X}=\mathrm{Br}$ and $\mathrm{Cl})$. Journal of Materials Chemistry C 7(31):9743-9747

[32] Yagmurcukardes M (2019) Monolayer fluoro-InSe: Formation of a thin monolayer via fluorination of InSe. Physical Review B 100(2):024108

[33] Castellanos-Gomez A, Poot M, Steele GA, van der Zant HS, Agrait N, Rubio-Bollinger G (2012) Elastic properties of freely suspended MoS2 nanosheets. Adv Mater 24(6):772-5

[34] Hung NT, Nugraha ART, Saito R (2018) Two-dimensional MoS2 electromechanical actuators. Journal of Physics D: Applied Physics 51(7):075306

[35] Sun S, Meng F, Xu Y, He J, Ni Y, Wang H (2019) Flexible, auxetic and strain-tunable two dimensional penta-X2C family as water splitting photocatalysts with high carrier mobility. Journal of Materials Chemistry A 7(13):7791-7799

[36] Hou B, Zhang Y, Zhang H, Shao H, Ma C, Zhang X, Chen Y, Xu K, Ni G, Zhu H (2020) Room Temperature Bound Excitons and Strain-Tunable Carrier Mobilities in Janus Monolayer TransitionMetal Dichalcogenides. J Phys Chem Lett 11(8):3116-3128

[37] Rudenko AN, Brener S, Katsnelson MI (2016) Intrinsic Charge Carrier Mobility in Single-Layer Black Phosphorus. Phys Rev Lett 116(24):246401

[38] Sun S, Meng F, Wang H, Wang H, Ni Y (2018) Novel two-dimensional semiconductor $\mathrm{SnP}_{3}$ : high stability, tunable bandgaps and high carrier mobility explored using first-principles calculations. Journal of Materials Chemistry A 6(25):11890-11897

[39] Zhang D, Hu S, Liu X, Chen Y, Xia Y, Wang H, Wang H, Ni Y (2020) Solar Cells Based on TwoDimensional $\mathrm{WTe}_{2} / \mathrm{PtXY}(\mathrm{X}, \mathrm{Y}=\mathrm{S}, \mathrm{Se})$ Heterostructures with High Photoelectric Conversion Efficiency and Low Power Consumption. ACS Applied Energy Materials 4(1):357-364

[40] Zhang LC, Qin G, Fang WZ, Cui HJ, Zheng QR, Yan QB, Su G (2016) Tinselenidene: a Twodimensional Auxetic Material with Ultralow Lattice Thermal Conductivity and Ultrahigh Hole Mobility. Sci Rep 6:19830 
[41] Bardeen J, Shockley W (1950) Deformation Potentials and Mobilities in Non-Polar Crystals. Physical Review 80(1):72-80

[42] Shen Y, Zhou Z (2008) Structural, electronic, and optical properties of ferroelectric $\mathrm{KTa}_{1 / 2} \mathrm{Nb}_{1 / 2} \mathrm{O}_{3}$ solid solutions. Journal of Applied Physics 103(7):074113

[43] Gajdoš M, Hummer K, Kresse G, Furthmüller J, Bechstedt F (2006) Linear optical properties in the projector-augmented wave methodology. Physical Review B 73(4):045112

[44] Lv L, Shen Y, Gao X, Liu J, Wu S, Ma Y, Wang X, Gong D, Zhou Z (2021) Strain engineering on the electrical properties and photocatalytic activity in gold sulfide monolayer. Applied Surface Science 546:149066

[45] Qiao M, Chen Y, Wang Y, Li Y (2018) The germanium telluride monolayer: a two dimensional semiconductor with high carrier mobility for photocatalytic water splitting. Journal of Materials Chemistry A 6(9):4119-4125

[46] Zhuang HL, Hennig RG (2013) Single-Layer Group-III Monochalcogenide Photocatalysts for Water Splitting. Chemistry of Materials 25(15):3232-3238 


\section{Supplementary Files}

This is a list of supplementary files associated with this preprint. Click to download.

- Graphicalabstract.docx

- supportinginformation.docx 